\title{
SIMULACIÓN Y EVALUACIÓN DE UNA PROPUESTA DE IMPLEMENTACIÓN DEL MÍNIMO VITAL DE AGUA POTABLE EN COLOMBIA
}

\author{
Jhon Alexander Méndez Sayago** \\ Johanna Mildred Méndez Sayago*** \\ Recibido: mayo 16 de 2010 \\ Aceptado: septiembre 30 de 2011
}

\section{RESUMEN}

Este artículo presenta la simulación y evaluación de la implementación del mínimo vital de agua potable para los usuarios de los estratos uno y dos, subsidiada a partir del incremento en las tarifas de los abonados de los estratos 5 y 6. La simulación exigió la estimación de funciones de demanda de agua, para determinar el impacto sobre el consumo de agua potable en los estratos 5 y 6, generados por los incrementos en el precio. Se determinó que existen ciudades como Neiva, Ibagué y Cúcuta en donde la propuesta es inviable, y otras con buenas posibilidades, como Bogotá, Manizales, Medellín, Cali, Bucaramanga y Santa Marta, en donde no se podría descartar la idea de entregar gratis hasta $10 \mathrm{~m} 3$ de agua potable por hogar al mes. En las ciudades de Popayán, Cartagena y Montería la propuesta tal vez solo sería factible para un mínimo vital de seis metros cúbicos por hogar al mes.

\section{PALABRAS CLAVE}

Mínimo vital de agua potable, simulación, demanda de agua, subsidios a la demanda de agua

\section{CLASIFICACIÓN JEL} Q25, H24

\section{CONTENIDO}

Introducción; 1. Derecho al agua en Colombia; 2. Mínimo vital de agua potable; 3. Estimaciones previas de la función de demanda de agua en Colombia; 4. Datos; 5. Estimación econométrica de la función de demanda de agua; 6. Evaluación de la propuesta de implementación del mínimo vital; 7. Conclusiones; Bibliografía.

" Este artículo de investigación es resultado del proyecto "Estimación del consumo básico de agua en Colombia", financiado mediante convocatoria interna de la facultad de Ciencias Sociales y Económicas de la Universidad del Valle, de Cali, Colombia, durante el año 2010. Surge por la necesidad de buscar una alternativa de financiamiento del mínimo vital de agua en Colombia.

** Ingeniero Civil, universidad Francisco de Paula Santander, Cúcuta, Colombia. Magíster en Economía del Medio Ambiente y de los Recursos Naturales, Universidad de los Andes, Bogotá, Colombia. Magíster en Economía, Universidad Javeriana, Bogotá, Colombia. Profesor Asistente de la Facultad de Ciencias Sociales y Económicas de la Universidad del Valle, Cali, Colombia. Miembro del grupo de investigación en Economía Regional y Ambiental (GERA), Universidad del Valle, clasificado como grupo C por COLCIENCIAS. Dirección postal: Ciudadela Universitaria Meléndez, Calle 13 No. 100-00, Edificio 387, Cali, Colombia. Correo electrónico: jhon.mendez@correounivalle.edu.co.

** Ingeniera de Producción Industrial, Universidad Francisco de Paula Santander, Cúcuta, Colombia. Magíster en Economía, Universidad Javeriana, Bogotá, Colombia. Profesora de la Facultad de Estudios a Distancia, Universidad EAN, Bogotá, Colombia. Dirección postal: Carrera 11 No.78-47 Bogotá, Colombia. Correo electrónico: jmmendez@correo.ean.edu.co. 


\section{SIMULATION AND EVALUATION OF AN IMPLEMENTATION PROPOSAL FOR THE MINIMUM VITAL OF DRINKING WATER IN COLOMBIA}

\section{ABSTRACT}

This article presents a simulation and evaluation of the minimum vital of drinking water implementation for the users of economic stratus 1 and 2, subsidized by the tariff increase of those individuals in stratus 5 and 6 . The simulation demanded the estimation of water demand functions in order to determine the impact over drinking water consumption that stratus 5 and 6 generated by the price increases. It was determined that cities such as Neiva, Ibague and Cucuta the proposal is not feasible, and in other cities with good implementation possibilities such as Bogota, Manizales, Medellin, Cali, Bucaramanga and Santa Marta where the idea of supplying the population with up to $10 \mathrm{~m} 3$ of drinking water per household per month. In the cities of Popayan, Cartagena and Monteria, the proposal probably won't be feasible for the required $6 \mathrm{~m} 3$ of drinking water per household per month.

\section{KEY WORDS}

Minimum vital of drinking water, simulation, water demand, subsidies for water demand.

\section{JEL CLASSIFICATION}

Q25, H24

\section{CONTENT}

Introduction; 1. Right on water in Colombia; 2. Minimum vital of drinking water; 3. Previous estimations of the water demand function in Colombia; 3. Data; 5. Econometric estimation for the water demand function; 6 . Evaluation of the proposal for the vital minimum of drinking water; 7 . Conclusions; Bibliography.

\section{SIMULAÇÃO E AVALIAÇÃO ECONÔMICA DE UMA PROPOSTA DE IMPLEMENTAÇÃO DO MÍNIMO VITAL DE AGUA POTÁVEL}

\section{RESUMO}

Este artigo apresenta a simulação e avaliação da implementação do mínimo vital de agua potável para os usuários de estratos 1 e 2, subsidiada desde o incremento nas tarifas dos abonados dos estratos 5 e 6 . A simulação exigiu a estimação de funções de agua para determinar o impacto sobre o consumo de agua potável nos estratos 5 e 6, gerados pelos incrementos no preço. Determinou-se que existem cidades como Neiva, Ibagué e Cúcuta onde a proposta e inviável, e outras com boas possibilidades como Bogotá, Manizales, Medellín, Cali, Bucaramanga e Santa Marta onde a ideia de entregar ate 10m3 de agua potável por lar ao mês não é descartada. Nas cidades de Popayán, Cartagena e Monteria a proposta tal vez só seria viável para um mínimo vital de seis metros cúbicos por lar ao mês.

\section{PALAVRAS-CHAVE}

Mínimo vital de agua potável, simulação, demanda de agua, subsídios à demanda de agua

\section{CLASSIFICAÇÃO JEL} Q25, H24

\section{CONTEÚDO}

Introdução; 1. Direito ao agua na Colômbia; 2. Mínimo vital de agua potável, 3. Estimações previas da função da demanda de agua na Colômbia; 4. Avaliação da proposta de implementação do mínimo vital; 7. Conclusões; Bibliografia. 


\section{INTRODUCCIÓN}

El agua hace parte fundamental del hombre, de su vida cotidiana y de sus valores más transcendentales. Sin ella, la supervivencia humana queda cuestionada. Es, por tanto, razonable que cada individuo se crea con derecho a una parte de ella.

El reconocimiento de que el agua es un bien sin el cual la vida humana no es posible impulsó en Colombia una iniciativa popular avalada por más de dos millones de firmas, denominada Referendo del Agua'. Según Alfaro y otros (2009) el objetivo del Referendo era lograr que en la Constitución, quedara consagrado el acceso al agua como un derecho fundamental, así todas las personas tendrían la posibilidad de un suministro mínimo en condiciones gratuitas, con independencia de su capacidad económica para pagar la tarifa del agua. Esto incluía el derecho a disponer de agua potable para beber y satisfacer las necesidades de higiene y preparación de alimentos; así el consumo humano se considera como prioritario frente a otros usos.

Sin embargo, el gobierno ${ }^{2}$ se opuso al texto original del referendo tras considerarlo regresivo e inconveniente. Según el gobierno, la propuesta podría conducir a un resultado contrario al de garantizar el líquido vital para todos los colombianos. Según Escobar (2009), uno de los argumentos esgrimidos para oponerse a la aprobación del proyecto era que en Colombia ya existía un mínimo vital gratuito para los estratos 1 y 2, configurado a través de las tarifas que subsidia el Estado para el consumo básico, lo que podría ser equiparado con un mínimo vital de $14 \mathrm{~m}^{3}$ por hogar para el estrato 1, y $8 \mathrm{~m}^{3}$ por hogar para el estrato 2. Según esa concepción, la propuesta de mínimo vital $\left(6-8 \mathrm{~m}^{3}\right.$

1 Proyecto de Ley Número 171 de 2008. En Colombia el referendo es un mecanismo de participación ciudadana, producto de la Reforma a la Constitución de 1991, por medio del cual los ciudadanos tienen la oportunidad de hacer saber a quienes han sido elegidos para tomar decisiones, los puntos de vista de la población acerca de un determinado asunto.

2 Para el año 2008 el presidente era Álvaro Uribe Vélez y el Ministro de Ambiente, Vivienda y Desarrollo Territorial era Juan Lozano Ramírez. por hogar) inferior al obtenido a través de los subsidios en las tarifas, solo beneficiaría a los estratos más altos. El gobierno tampoco estaba de acuerdo con otras disposiciones del Referendo del agua, como la prohibición de prestación del servicio para empresas privadas, y la asignación de la conservación de las fuentes de agua a ciertas comunidades étnicas o nativas. Todo lo anterior contribuyó a que la propuesta se hundiera en el Congreso de la República.

Concentrándose solo en la propuesta de mínimo vital gratuito de agua potable del Referendo del Agua, es debatible desde una perspectiva económica que esta disposición deba tener carácter universal, ya que en la práctica, los hogares de mayores ingresos, por su capacidad de pago, tienen asegurado su acceso al mínimo vital de agua potable

En este sentido, ya que los recursos del Estado son limitados, habría que desfinanciar de manera parcial la expansión de la cobertura, para destinar recursos a la financiación del mínimo vital, a pesar de que este también favorece a los más ricos. Además, habría que tener en cuenta que según el esquema tarifario de Colombia, definido por la Ley 142 de1994, los estratos 5 y 6 tienen factores de ajuste de tarifas del $120 \%$ que ayudan a subsidiar las tarifas de los estratos 1, 2 y 3. Con la propuesta original del Referendo del Agua, este recaudo sería erosionado, porque ya no se les cobraría a los estratos 5 y 6 la cantidad correspondiente al mínimo vital gratuito.

Por otra parte, también es discutible el argumento del gobierno de que gracias al esquema tarifario actual, los usuarios de los estratos 1 y 2 tengan acceso a un mínimo vital de agua. A un hogar del estrato 1 no se le suministran $14 \mathrm{~m}^{3}$ sin cobro (ni $8 \mathrm{~m}^{3}$ al estrato 2), porque el usuario debe pagar la factura resultante después de aplicar el subsidio, independiente de que su consumo sea inferior al supuesto mínimo vital, algo imposible para muchos hogares de ingresos bajos, razón por la cual, según los defensores del Referendo del Agua, existen cerca 
de 400 mil suscriptores (1.6 millones de personas) desconectados al año en todo el país ${ }^{3}$.

Esa discusión lleva a proponer en esta investigación la evaluación de una propuesta de mínimo vital de agua potable para los suscriptores de los estratos 1 y 2 de las principales ciudades de Colombia. Esta propuesta de mínimo vital modifica los factores de ajuste de las tarifas del sistema vigente de subsidios cruzados, para que los usuarios de los estratos altos (5 y 6) sean quienes financien el mínimo vital de agua de los estratos 1 y 2, sin incrementar el déficit actual del sistema.

La evaluación de la propuesta requirió la simulación del impacto financiero de la entrega del mínimo vital gratuito para los estratos 1 y 2, y la medición de la caída en el consumo de agua de los estratos 5 y 6 como producto del aumento en las tarifas del agua. La segunda exigencia se superó con la estimación econométrica de una función de demanda de agua; se utiliza un conjunto de datos panel. La simulación permitió determinar en cuáles de las principales ciudades de Colombia es posible que los estratos 5 y 6 subsidien $8 \mathrm{~m}^{3} \circ 6 \mathrm{~m}^{3}$ de mínimo vital gratuito de agua por hogar, y en cuáles esto solo sería posible con aportes del gobierno.

Este artículo está estructurado de la siguiente forma: en primer lugar se explora la posible existencia del derecho al agua en Colombia, después se realiza una revisión bibliográfica para la conceptualización del mínimo vital de agua y la determinación de la cantidad de agua potable que les garantiza a los hogares unas condiciones de vida digna. Luego se presentan los resultados de estimaciones previas de funciones de demanda de agua en Colombia para tener alguna referencia de la elasticidad precio del agua. Por último, se muestran los datos disponibles, se precisan los aspectos metodológicos y se dan a conocer los resultados de la evaluación de la propuesta de mínimo vital gratuito de agua y las conclusiones.

Según encuesta de satisfacción de usuarios de la SSPD, 2006, citado por Suarez y Cardona (2008, p. 7).

\section{DERECHO AL AGUA EN COLOMBIA}

El agua es un bien social y un bien económico que debe distribuirse de forma equitativa para satisfacer, en primera medida, las necesidades humanas básicas (Díaz y otros, 2009). Dentro de los recursos naturales, el agua es el principal de todos, por ser imprescindible para la vida y la salud de las personas (Muñoz, 2009). Entonces el derecho al agua refleja una realidad innegable: sin agua no hay vida.

No hay nada que pueda sustituir el agua: sin ella perecen los seres humanos y otros organismos vivos. El ser humano no puede estar sin beberla más de cinco o seis días sin poner en peligro su vida (PNUD, 2006), porque requiere para su mantenimiento, el suministro de una cantidad similar a la que ha perdido de agua para realizar las funciones biológicas básicas.

Según Díaz y otros (2009, p. 91),

... sin agua, los agricultores no pueden cultivar los alimentos; se afectan los sectores pecuario, piscícola, industrial y algunos de servicios, como el turismo y la recreación (las piscinas y los juegos acuáticos). La seguridad en el abastecimiento de agua es indispensable para el desarrollo de los pueblos, porque, aparte de la importancia para la supervivencia, propicia el desarrollo productivo, de manera que ayuda a la reducción de la pobreza.

De acuerdo con Valencia (2008, p. 4), ... la carencia del agua y sobre todo del agua con calidad, tiene por el contrario grandes implicaciones negativas en la vida humana; por ejemplo, la más visible, es el número de muertes causadas por las enfermedades asociadas al agua; siendo los niños la población más afectada.

El reconocimiento de la importancia que tiene el agua para la vida, y de la adversidad que afrontan quienes no tienen acceso a ella, en las condiciones adecuadas, motivó a que en octubre del año 2008 se presentara al Congreso de la República de Co- 
lombia el Referendo del Agua. La propuesta tenía como propósitos:

1. Que el acceso al agua sea un derecho fundamental.

2. Garantizar un mínimo vital gratuito para los hogares colombianos.

3. Que los servicios públicos de acueducto y alcantarillado no se privaticen y sean prestados por entidades públicas o por acueductos comunitarios sin ánimo de lucro.

4. Que se garantice la efectiva conservación del medioambiente necesario para que haya agua abundante y limpia.

Incorporar en la Constitución el derecho al agua como derecho fundamental es un hecho transcendental para la población más desfavorecida. Al respecto Smets (2006a, p. 14) argumenta

... el reconocimiento explícito del derecho al agua como derecho fundamental constituye una acción importante que tiene un valor simbólico y jurídico elevado. Este reconocimiento prueba la importancia que los poderes públicos atribuyen al agua para la salud y la consideración que tienen por los deseos de los usuarios que no tiene acceso al agua potable.

Sin embargo, por las razones ya expuestas, el Congreso decidió negar por completo las modificaciones propuestas por el Referendo en su texto original, y el Referendo del Agua se hundió en el Congreso de la República.

En la misma línea del Referendo del Agua, en el año 2010, ante propuesta presentada por Bolivia y que contó con voto favorable de Colombia ${ }^{4}$, la Asamblea General de las Naciones Unidas (ONU, 2010, p. 3), en Resolución A/64/L.63/Rev.1 decidió declarar el acceso al agua potable como derecho humano: "Declara el derecho al agua potable y el saneamiento

\footnotetext{
$4 \quad$ La resolución contó con 122 votos a favor, de los 192 miembros de las Naciones Unidas, no hubo votos en contra, 41 países se abstuvieron, ante todo países desarrollados como estados Unidos, Reino Unido y Australia. Las naciones en vías de desarrollo en general votaron a favor.
}

como un derecho humano esencial para el pleno disfrute de la vida $y$ de todos los derechos humanos".

No obstante, como afirma Araújo (2009), aunque exista una progresiva positivación interna de los derechos humanos en los derechos fundamentales, no pueden ser entendidos como la misma cosa, porque la efectividad de cada uno es diferenciada. Así que para los millones de colombianos que no disponen de agua potable y saneamiento básico, la resolución A/64/L.63/Rev.1 no implica un cambio en las políticas oficiales. Esto se debe a que la resolución no convierte el agua en un derecho fundamental de los colombianos, tutelable y exigible, porque no es vinculante en el marco del derecho internacional, así que no se obliga a los Estados a cumplirla, solo los exhorta para que proporcionen recursos financieros, a fin de intensificar los esfuerzos por suministrar a toda la población un acceso económico al agua potable y el saneamiento.

\section{MÍNIMO VITAL DE AGUA POTABLE}

¿Cuánta agua debe utilizar cada persona para tener unas condiciones de vida digna?

Esta es una pregunta compleja, ya que existen varios factores que determinan el mínimo vital de agua potable (variables geográficas, históricas, culturales), así que para el mínimo vital no se puede encontrar una medida universal, por el contrario, deben estudiarse las características de las comunidades a las que se quiere suministrar este mínimo para poder realizar los cálculos de manera adecuada. Como argumenta Valencia (2008, p. 4) "No es lo mismo hacer un cálculo para una población en un territorio donde históricamente se carece de agua, a otro donde de manera abundante siempre la han tenido; en este caso la cultura determina el nivel de consumo y sus necesidades".

A manera de referencia puede insinuarse la recomendación de Howard y Bartram (2003), quienes en un documento de la Organización Mundial de la Salud (OMS), relacionan el nivel de servicio de agua con la higiene, a través de la cantidad de agua disponible. En el documento citado no se define un 
volumen mínimo vital, sino un nivel de afectación a la salud en función de las condiciones de acceso al agua. Los requerimientos mínimos de agua se presentan en la tabla 1. Obsérvese que según la OMS la cifra de 50 litros por hombre día (lhd) cubre los requerimientos básicos de higiene y consumo, necesidades vitales como bañarse, cocinar y otras, así que esta cifra es una buena guía.

Académicos y líderes políticos que se preocupan por el mismo tema hablan de cifras similares, por ejemplo, Martínez (2004), propuso en el Foro Social Mundial de 2004, un piso de dignidad (free lifeline) para las zonas urbanas de 50 lhd; Valencia (2008) referencia la propuesta de 60 lhd de Alexandra Sandton, en el 2002, en la cumbre oficial de las Naciones Unidas en Johannesburgo.

Valencia (2008) propone una forma fácil de hacer el cálculo; la idea consiste en establecer cuál es el consumo de agua por actividad: para beber 5 litros, para saneamiento 25 litros, para higiene 15 litros, para cocinar 10 litros y para otros usos 5 litros. Su suma conduce a la cifra de 60 lhd.
Smets (2006b) manifiesta que en la región de Flandes (Bélgica) en 1996, se adoptó un decreto que estipula que cada abonado tiene derecho a un suministro mínimo e ininterrumpido de electricidad, gas y agua para uso doméstico con el fin de poder llevar una vida humana de acuerdo con el nivel de vida vigente. Según Valencia (2008) en Bélgica se estableció una cuota de servicio fija, que da derecho a consumir un mínimo vital de 40 lhd gratuitos por persona, y en el País Vasco (España) se estableció un mínimo exento del canon ecológico de 130 lhd.

Davidson (2009) expone que en Sudáfrica, en el año 2000, el gobierno nacional anunció que habría una política de "acceso gratuito al agua" a partir del 2001, suministrada por las autoridades municipales y financiada en forma parcial por el gobierno nacional. La promesa se hizo efectiva y la cantidad asignada fue de 6.000 litros por hogar por mes, calculados con base en una estimación de 25 litros por persona por día en hogar de ocho personas

En el año 2009 Medellín se convirtió en la primera ciudad en Colombia (y única en la actualidad)

Tabla 1. Resumen de la exigencia del nivel servicio de agua para promover la salud

\begin{tabular}{|c|c|c|c|}
\hline $\begin{array}{l}\text { Descripción del } \\
\text { nivel de servicio }\end{array}$ & $\begin{array}{c}\text { Medida de tiempo o distancia } \\
\text { de acceso }\end{array}$ & Cantidad de agua disponible & Nivel de afectación a la salud \\
\hline Sin acceso & $\begin{array}{l}\text { Más de } 1000 \mathrm{~m} \text { o } 30 \mathrm{mi}- \\
\text { nutos de tiempo total de } \\
\text { aprovisionamiento. }\end{array}$ & $\begin{array}{l}\text { Muy Baja (por lo general menos de } \\
5 \text { lhd) }\end{array}$ & $\begin{array}{l}\text { Muy alto puesto que la higiene } \\
\text { no está garantizada y las nece- } \\
\text { sidades de consumo pueden } \\
\text { estar en riesgo. La calidad es } \\
\text { difícil de garantizar. }\end{array}$ \\
\hline Acceso básico & $\begin{array}{l}\text { Entre } 100 \text { y } 1000 \text { m ( } 5 \text { a } 30 \\
\text { minutos de tiempo total de } \\
\text { aprovisionamiento). }\end{array}$ & $\begin{array}{l}\text { Baja. El promedio pocas veces } \\
\text { excede } 20 \text { lhd. Los usos de aseo } \\
\text { personal y lavado de ropa pueden } \\
\text { satisfacerse en la fuente de agua } \\
\text { con volúmenes adicionales de agua. }\end{array}$ & $\begin{array}{l}\text { Medio. Puede que no se satis- } \\
\text { fagan todos los requerimien- } \\
\text { tos. La calidad es difícil de } \\
\text { garantizar. }\end{array}$ \\
\hline $\begin{array}{l}\text { Acceso } \\
\text { intermedio }\end{array}$ & $\begin{array}{l}\text { En el predio a través de } \\
\text { una pluma en la vivienda } \\
\text { o en el lote. }\end{array}$ & $\begin{array}{l}\text { Media. Por lo general alrededor de } \\
50 \text { lhd. }\end{array}$ & $\begin{array}{l}\text { Bajo. La mayoría de requeri- } \\
\text { mientos de higiene y consumo } \\
\text { son satisfechos. La calidad es } \\
\text { garantizada más fácil. }\end{array}$ \\
\hline Acceso óptimo & $\begin{array}{l}\text { El agua es conducida al } \\
\text { interior de la vivienda a } \\
\text { través de múltiples plumas. }\end{array}$ & $\begin{array}{l}\text { Varía en forma significativa pero } \\
\text { casi siempre es superior a } 100 \text { lhd } \\
\text { y puede llegar a } 300 \text { lhd. }\end{array}$ & $\begin{array}{l}\text { Muy Bajo. Todos los usos } \\
\text { son satisfechos, la calidad es } \\
\text { garantizada. }\end{array}$ \\
\hline
\end{tabular}

Fuente: Howard y Bartram (2003, p. 3) 
en tener un programa de mínimo vital de agua potable. Según Marín (2009) este mínimo corresponde a 2,5 $\mathrm{m}^{3}$ de agua potable, que cada uno de los hogares más vulnerables de la ciudad recibe cada mes por persona. Entonces un hogar conformado por cuatro personas, que es el promedio Nacional, tiene derecho a $10 \mathrm{~m}^{3}$ de agua potable.

Como lo manifiesta Valencia (2008), existen diversas posiciones sobre las cantidades del mínimo vital de agua. Afirma que ello se debe a las diferencias culturales, climáticas, sociales y económicas entre las regiones del mundo. Esto refuerza la idea de que se debe estudiar cada región antes de establecer un consumo mínimo vital de agua potable.

\section{ESTIMACIONES PREVIAS DE LA FUNCIÓN DE DEMANDA DE AGUA EN COLOMBIA}

La evaluación de una propuesta de implementación del mínimo vital de agua en Colombia, subsidiada por los estratos socioeconómicos altos (estratos 5 y 6), requiere la estimación de la una función de demanda de agua, en particular, para determinar los efectos sobre el consumo de los abonados de esos estratos ante al cobro de una tarifa más alta, y así, lograr simular la propuesta. Por esta razón, a continuación se presenta un resumen de las estrategias habituales para la especificación y estimación de las funciones de demanda de agua.

Uno de los principales trabajos realizados en Colombia corresponde a Junca (2000), en el cual se estiman funciones de demanda de agua, para determinar el rango de consumo básico de agua potable subsidiable para el sector residencial, a partir de consumos históricos y tarifas para las ciudades de Medellín, Bogotá y Cali. La especificación de la función de demanda obedece a que se parte del modelo de ajuste parcial, para justificar la incorporación de los hábitos de consumo y se opta por relacionar las cantidades consumidas y los precios, a través de una curva de demanda de elasticidad constante:

$$
\begin{aligned}
\log Q_{t, i=} & \log C_{t, i}+\beta_{1} \log \left(P_{t-1, i}\right) \\
& +\beta_{2} \log \left(Q_{t-1, i}\right)+\varepsilon_{t, i} \quad i=1 \ldots 6
\end{aligned}
$$

Donde:

Q: Cantidad promedio de agua consumida al mes por los usuarios del estrato $i\left(\mathrm{en}^{3}\right)$

C: Consumo básico del usuario del estrato $i$.

P: Tarifa promedio para el usuario del estrato $i$. (pesos por $\mathrm{m}^{3}$, a precios constantes de 1998).

$\beta_{1}$ : Elasticidad precio de la demanda de agua.

t: Periodo de tiempo (mes).

є: Error idiosincrásico

La estimación se hizo por mínimos cuadrados ordinarios, con la información de las series de tiempo de tarifas y consumos, para cada uno de los estratos. En la estimación se desaprovechó el hecho de que se contara con información del tipo de datos panel, así que también se ignoraron las técnicas de estimación propias de este conjunto de datos.

Todas las elasticidades precio de la demanda estimadas presentaron el signo esperado (negativo) y todas resultaron estadísticamente significativas. Las elasticidades estimadas aparecen en las tabla 2. Junca (2000, p. 28) resalta que el valor absoluto de las elasticidades estimadas tiende a aumentar a medida que el nivel de ingresos de las familias es superior". Argumenta que "este resultado es consistente con lo esperado, dado que los estratos bajos ajustan los niveles al mínimo esperado, es decir están sobre su consumo básico". Las familias más pobres no tienen margen de reacción ante los incrementos en los precios de los servicios; pero a medida que los niveles de ingresos aumentan, se incrementa también este margen, sin renunciar a la atención de las necesidades básicas.

Por último, se recomienda la adopción como consumo básico subsidiable de agua potable la cantidad correspondiente a $16 \mathrm{~m}^{3}$ por hogar al mes. 
Tabla 2. Elasticidades precio de la demanda estimadas por Junca (2000)

\begin{tabular}{|c|c|c|c|}
\hline Estrato & EAAB & EPM & EMCALI \\
\hline 1 & $-0,137$ & $-0,040$ & $-0,235$ \\
\hline 2 & $-0,146$ & $-0,196$ & $-0,247$ \\
\hline 3 & $-0,195$ & $-0,193$ & $-0,396$ \\
\hline 4 & $-0,273$ & $-0,087$ & $-0,230$ \\
\hline 5 & $-0,273$ & $-0,335$ & $-0,362$ \\
\hline 6 & $-0,390$ & $-0,332$ & $-0,195$ \\
\hline
\end{tabular}

Fuente: Junca (2002, p. 29)

Un estudio realizado por la Comisión de Regulación de Agua Potable y Saneamiento Básico (CRA, 2008), el cual tenía como objetivo determinar el rango de consumo básico para el servicio de acueducto, con el propósito de reformar el vigente, utilizó la información de consumos y tarifas para el período 1994-2008 de las ciudades de Bogotá, Medellín, Cali y Barranquilla para estimar, con ayuda de modelos econométricos de datos panel, la elasticidad precio de la demanda de agua potable de los estratos 1, 2 y 3, los únicos relevantes para la política de otorgamiento de subsidios.

Las elasticidades precio de la demanda estimadas por CRA (2008) para los estratos uno, dos y tres fueron en su orden: $-0.118 \%,-0.179 \%$ y $-0.155 \%$. Ellos concluyen que a medida que se incrementa el estrato, la sensibilidad de los cambios en el consumo ante cambios en el precio aumenta, porque la tarifa es más alta, a medida que aumenta el estrato, porque el subsidio que reciben es menor. Sin embargo, esta afirmación no se ajusta con exactitud a los resultados, ya que aunque el estrato 3 paga el precio más alto por $\mathrm{m}^{3}$ por recibir un subsidio menor (15\%), tiene una elasticidad precio más pequeña (en valor absoluto) que el estrato dos, que recibe un subsidio del $40 \%$.

Por otra parte, ellos concluyen que el rango de $20 \mathrm{~m}^{3}$ por suscriptor al mes de consumo básico se encuentra sobreestimado, y proponen diferenciar el consumo por rangos climáticos, de modo que las ciudades de clima frío (altitud mayor a 2000 msnm) tengan un consumo básico de $17 \mathrm{~m}^{3}$ por suscriptor al mes, las ciudades de clima templado (altitud entre 1000 y 2000 msnm) tengan un consumo básico de $18 \mathrm{~m}^{3}$ por suscriptor al mes, y las ciudades de clima cálido (altitud menor a 1000 msnm) tengan un consumo básico de $19 \mathrm{~m}^{3}$ por suscriptor al mes.

Medina y Morales (2007) utilizaron el método de elección discreta continua propuesto por Hewitt y Hanemann (1995) para superar las dificultades econométricas que según ellos, imponen los bloques de precios de las tarifas de los servicios públicos de acueducto y alcantarillado en Colombia. Los autores argumentan que, cuando la estructura de precios de un determinado bien es del tipo de bloques crecientes, y el precio marginal aumenta en la medida que aumenta el consumo, se presenta un problema de simultaneidad, dado que el precio y el bloque de consumo se determinan en forma simultánea. La simultaneidad tiene como consecuencia que de la estimación de la ecuación de demanda por mínimos cuadrados ordinarios se obtengan estimadores insesgados e inconsistentes por la endogeneidad de la variable precio.

Medina y Morales (2007) señalan algunas limitaciones de la técnica de variables instrumentales (VI), la más común para superar el problema de la endogeneidad: con el método de VI solo se pueden estimar elasticidades condicionales al bloque observado de consumo; cuando el consumo está muy cercano a un salto de bloque no es claro cuál valor de precio marginal debe asignársele y, por último, el efecto de los cambios en la estructura de precio o cambio en la cantidad de bloques que enfrenta cada consumidor no puede ser evaluado como un elemento de la estructura de precio. Esos argumentos hacen que sea preferible la estimación por el método de elección discreta continua.

La elasticidad precio de la demanda estimada mediante simulaciones fue de $-0,3$. Así que concluyen que si se registrase un incremento o decremento 
en el precio del agua, equivalente al doble del precio actual, el consumo promedio solo se incrementaría o reduciría en $30 \%$, respectivamente.

\section{DATOS}

Los datos para el sector urbano del consumo promedio por hogar para los diferentes estratos, las tarifas para el consumo básico, consumo complementario y consumo suntuario de los diferentes estratos, así como el número de usuarios por estrato fueron extraídos de la página web del Sistema Único de Información (SUI) ${ }^{5}$. La estimación econométrica de la función de demanda de agua se hizo con base en los registros desde enero del año 2003 a diciembre del año 2008, para 21 ciudades colombianas: Armenia, Barranquilla, Bogotá, Bucaramanga, Cali, Cartagena, Cúcuta, Florencia, Manizales, Pereira, Tunja, Pasto, Villavicencio, Ibagué, Medellín, Montería, Neiva, Popayán, Santa Marta, Sincelejo, Valledupar. Con la información disponible, fue posible organizar para cada estrato, un conjunto de datos panel desbalanceado ${ }^{6}$.

La tabla 3, que contiene las estadísticas descriptivas del consumo de agua potable por estrato, muestra que el consumo de agua promedio es mayor a medida que aumenta el estrato. La dispersión del consumo presenta el mismo comportamiento. La evolución del consumo promedio de agua por estrato para cada una de las ciudades que se muestra en el anexo A, evidencia que en la mayoría de los casos se cumple que el consumo se reduce con el paso de los años. Este hecho obedece al aumento paulatino de las tarifas de agua potable durante los últimos años y, posiblemente también, al efecto de la instalación de dispositivos hidráulicos de ahorro en duchas e instalaciones sanitarias, así como lavadoras de ropa que utilizan menor cantidad de agua para su operación.

5 El link de esta página web es: http://www.sui.gov.co/SUIWeb/ logon.jsp.

6 El lector interesado puede solicitar por email la base de datos que se empleo para obtener los resultados que presentan en este artículo.
Tabla 3. Estadísticas descriptivas del consumo de agua potable por estrato, 2003-208

\begin{tabular}{|l|c|c|c|c|c|c|}
\hline & \multicolumn{5}{|c|}{ Consumo (en $\mathrm{m}^{3}$ por hogar al mes) } \\
\cline { 2 - 7 } & $\begin{array}{c}\text { Estrato } \\
1\end{array}$ & $\begin{array}{c}\text { Estrato } \\
2\end{array}$ & $\begin{array}{c}\text { Estrato } \\
3\end{array}$ & $\begin{array}{c}\text { Estrato } \\
4\end{array}$ & $\begin{array}{c}\text { Estrato } \\
5\end{array}$ & $\begin{array}{c}\text { Estrato } \\
6\end{array}$ \\
\hline Mean & 15,27 & 17,56 & 18,50 & 20,13 & 21,62 & 26,94 \\
\hline Median & 14,59 & 16,73 & 18,34 & 19,59 & 20,98 & 25,46 \\
\hline Maximum & 28,58 & 31,22 & 28,45 & 31,83 & 36,98 & 45,57 \\
\hline Minimum & 8,54 & 9,9 & 10,30 & 10,33 & 12,04 & 12,23 \\
\hline Std. Dev. & 3,850 & 4,199 & 3,891 & 4,441 & 5,415 & 6,971 \\
\hline $\begin{array}{c}\text { Observa- } \\
\text { ciones }\end{array}$ & 998 & 998 & 995 & 994 & 944 & 907 \\
\hline
\end{tabular}

Fuente: elaboración propia

\section{ESTIMACIÓN ECONOMÉTRICA DE LA FUNCIÓN DE DEMANDA DE AGUA}

Primero se trató de estimar la función de demanda mediante un modelo de elasticidad constante para todos los estratos:

$$
\log Q_{i, t}^{s}=\beta_{i, 1}^{s}+\beta_{2}^{s} \log \left(p_{i, t-1}^{s}\right)+\varepsilon_{i, t}
$$

Donde:

$\log Q_{i, t}^{s}$ : Logaritmo natural del consumo promedio de agua para el estrato $s$ de la ciudad de la ciudad $i$ durante el periodo $t$.

$\log \left(p_{i, t-1}^{s}\right)$ : Logaritmo natural del precio real del agua en el estrato s de la ciudad de la ciudad $i$ rezagado un periodo.

S: $\quad$ Número de estratos, 6.

N: Total de ciudades de la muestra, 21.

$T: \quad$ Número de meses en los que se tiene registro del consumo y el precio, 72 .

$\beta_{i, 1}^{s}$ : Intercepto cambiante entre los individuos, que para este caso específico representa el consumo básico del estrato s en la ciudad $i$.

$\beta_{2}^{s}$ : Elasticidad precio de la demanda de agua para cada estrato. 


$$
\beta_{i, 1}^{s}=\beta_{2}^{s}+a_{i}^{s}
$$

$a_{i}^{s}$ : Efecto no observado para el estrato $s$ de la ciudad $i$. Recoge toda la heterogeneidad individual. Permite que el consumo básico de un mismo estrato difiera entre las distintas ciudades, cosa que puede ocurrir por efecto de la temperatura ${ }^{7}$.

$\beta_{1}^{s}$ : Consumo básico promedio por estrato.

La especificación de la ecuación (2) ignora los hábitos de consumo al no incluir rezagos de la variable dependiente. La omisión se debe a que para la simulación solo es importante el efecto de los incrementos en la variable precio, por tanto solo es importante estimar la elasticidad precio de la demanda. Incluir el consumo rezagado hace que se viole el supuesto de exogeneidad estricta de los residuales en el modelo de efectos fijos, lo que conduciría a estimadores que, además de sesgados, serían inconsistentes. Esto obligaría a estimar el modelo con la técnica de variables instrumentales (p. ej. Estimador de Hsiao) o el método generalizado de momentos (p. ej. Arellano Bond). Sin embargo, para datos panel desbalanceados, y con $\mathrm{T}>\mathrm{N}$, la complicación adicional supera las expectativas sobre la bondad de los estimadores ${ }^{8}$.

Por otra parte, con la especificación (2) se descarta la endogeneidad por simultaneidad, como lo sugieren Medina y Morales (2007), ya que no es posible que el consumo actual afecte las tarifas del agua en el pasado. No obstante, la especificación de la ecuación (2) obedece a que las personas no pueden tomar las decisiones sobre el consumo de agua potable con base en el precio del mismo período, porque este es desconocido para el usuario,

7 De hecho aunque se incluyó la variable temperatura en la ecuación (2), la variable no resultó significativa. Esto puede atribuírsele a que su impacto sea captado por el efecto fijo, ya que la temperatura es una variable que para una misma ciudad, presenta poca variación en el tiempo.

8 La omisión de la variable consumo rezagado en la ecuación (2) puede generar autocorrelación, por tanto, para las pruebas de hipótesis se utilizaron errores estándar robustos. que solo conoce la tarifa cuando recibe la factura, en el siguiente mes. Así que los abonados al servicio de acueducto tiene toman las decisiones de consumo actual con fundamento en el precio del periodo anterior.

Para los estratos uno hasta cuatro, la especificación (2) de la ecuación de demanda no resultó viable, porque los resultados de las estimaciones evidenciaron que no es posible rechazar la hipótesis nula, de que las elasticidades precio de la demanda de esos cuatro estratos es cero. Por lo tanto, para esos cuatro estratos se estimó un modelo ingenuo de la forma:

$$
Q_{i, t}^{s}=\tilde{Q}_{1}^{s}+a_{i}^{s}+\varepsilon_{i, t}
$$

Donde:

$Q_{i, t}^{s}$ : Consumo promedio de agua potable por estrato s y ciudad $i$.

$\tilde{Q}_{1}^{s}$ : Consumo promedio de las 21 ciudades (promedio Nacional)

$a_{i}^{s}$ : Componente de la heterogeneidad individual sobre el consumo básico de agua potable, por estrato s y ciudad $i$.

La estimación de los modelos con la especificación (2) se llevó a cabo mediante la técnica de efectos aleatorios. La estimación de los modelos ingenuos se efectuó bajo el modelo de variable dummy, el cual para el caso resulta ser el promedio individual. Las estimaciones se muestran en la tabla 4 . Con $=5 \%$ se rechaza la hipótesis nula de elasticidad precio de la demanda es cero para los estratos 5 y 6 . Los test de Hausman que aparecen en las tablas 5 y 6 demuestran que no hay diferencias significativas entre los estimadores de efectos aleatorios y efectos fijos, por lo tanto, se descarta la posible correlación entre el efecto no observado y la variable precio, así que se prefiere la estimación por efectos aleatorios. En el anexo B se encuentra el componente de heterogeneidad individual para cada uno de los seis estratos. 
Tabla 4. Resultados de la estimación de las ecuaciones de demanda

\begin{tabular}{|c|c|c|c|c|c|c|}
\hline & Coeficiente & Estimador & Desv. Est. & Estadístico $t$ & $p$-valor & R cuadrado \\
\hline \multirow{2}{*}{ Estrato 1} & $\tilde{Q}_{1}^{1}$ & 15,27737 & $6,68 \mathrm{E}-17$ & $2,29 \mathrm{E}+17$ & 0,0000 & \multirow{2}{*}{0.87} \\
\hline & - & - & - & - & - & \\
\hline \multirow{2}{*}{ Estrato 2} & $\tilde{Q}_{1}^{2}$ & 17,5684 & $1,05 \mathrm{E}-16$ & $1,68 \mathrm{E}+17$ & 0,0000 & \multirow{2}{*}{0.90} \\
\hline & - & - & - & - & - & \\
\hline \multirow{2}{*}{ Estrato 3} & $\tilde{Q}_{1}^{3}$ & 18,5035 & $1,05 E-16$ & $1,76 \mathrm{E}+17$ & 0,0000 & \multirow{2}{*}{0.89} \\
\hline & - & - & - & - & - & \\
\hline \multirow{2}{*}{ Estrato 4} & $\tilde{Q}_{1}^{4}$ & 20,13152 & $6,79 \mathrm{E}-17$ & $2,97 \mathrm{E}+17$ & 0,0000 & \multirow{2}{*}{0.90} \\
\hline & - & - & - & - & - & \\
\hline \multirow{2}{*}{ Estrato 5} & $\tilde{\beta}_{1}^{5}$ & 3,903465 & 0,272102 & 14,34561 & 0,0000 & \multirow{2}{*}{0.91} \\
\hline & $\tilde{\beta}_{2}^{5}$ & $-0,129947$ & 0,041733 & $-3,113736$ & 0,0019 & \\
\hline \multirow{2}{*}{ Estrato 6} & $\tilde{\beta}_{1}^{6}$ & 4,046438 & 0,360703 & 11,21819 & 0,0000 & \multirow[b]{2}{*}{0.88} \\
\hline & $\tilde{\beta}_{2}^{6}$ & $-0,117459$ & 0,052809 & $-2,224213$ & 0,0264 & \\
\hline
\end{tabular}

Fuente: elaboración propia

Tabla 5. Test de Hausman (estrato 5)

\begin{tabular}{|l|r|r|r|}
\hline \begin{tabular}{l} 
Correlated Random Effects - Hausman Test \\
Equation: EQ05 \\
Test cross-section random effects \\
\hline Test Summary
\end{tabular}$\quad \begin{array}{r}\text { Chi-Sq. } \\
\text { Statistic }\end{array}$ & Chi-Sq. d.f. & Prob. \\
\hline Cross-section random & 0.023903 & 1 & $0.8771^{*}$ \\
\hline
\end{tabular}

*Se acepta la Ho: No hay diferencia entre $\hat{E F}$ y $\hat{E A}$ Fuente: elaboración propia

Tabla 6. Test de Hausman (estrato 6)

\begin{tabular}{|lllll|}
\hline $\begin{array}{l}\text { Correlated Random Effects - Hausman Test } \\
\text { Equation: EQ06 } \\
\text { Test cross-section random effects }\end{array}$ & & & \\
\hline Test Summary & $\begin{array}{r}\text { Chi-Sq. } \\
\text { Statistic }\end{array}$ & Chi-Sq. d.f. & Prob. \\
\hline Cross-section random & 0.014276 & 1 & $0.9049^{*}$ \\
\hline
\end{tabular}

* Se acepta la Ho: No hay diferencia entre $\hat{E F}$ y $\hat{E A}$

Fuente: elaboración propia 


\section{EVALUACIÓN DE LA PROPUESTA DE IMPLEMENTACIÓN DEL MÍNIMO VITAL}

La propuesta de implementación del mínimo vital formulada en este artículo consiste en conceder de forma gratuita cierta cantidad de m3 de agua potable a los estratos uno y dos de las principales ciudades de Colombia, sin profundizar el déficit actual del sistema. Para que esto sea posible, los abonados de los estratos 5 y 6 deben subsidiar el mínimo vital, así que se requiere un aumento en las tarifas. Para los estratos tres y cuatro se conserva la metodología tarifaria vigente.

En ese contexto, se evalúa la viabilidad de otorgar una cantidad de $10 \mathrm{~m} 3,8 \mathrm{~m} 3$ y $6 \mathrm{~m} 3$ de agua potable a los usuarios de los estratos uno y dos, que representarían el mínimo vital. En tales condiciones, estos usuarios solo tendrían que pagar por el excedente de consumo de agua:

\section{Excedente $=Q_{i, t}^{s}-$ mínimo vital $s=1,2$}

La Tabla 7 muestra los consumos promedios estimados para los cuatro primeros estratos, calculados a partir del modelo ingenuo (ecuación 4), para las 13 ciudades $^{9}$ para las cuales se evaluó la propuesta.

La simulación se hizo con base en la cantidad de usuarios y las tarifas de diciembre del año 2009. La tabla 8 muestra el número de usuarios por estrato y total para cada ciudad. La tabla 9 muestra el porcentaje de beneficiarios del mínimo vital (usuarios de estratos uno y dos), los subsidiadores (usuarios de estratos cinco y seis) y la relación entre los dos. Esta relación es fundamental en la viabilidad de la implementación del mínimo vital.

En la tabla 10 aparecen los resultados de la simulación de la propuesta para las diferentes cantidades de $\mathrm{m}^{3}$ de agua correspondientes al mínimo vital. Se muestran las variaciones en el precio real del agua para el estrato 5. Esta propuesta conserva las

9 Ocho ciudades no reportaron al SUI información reciente, razón por la cual fue necesario excluirlas para realizar la simulación de la propuesta de mínimo vital. relaciones de precios entre las tarifas de los estratos 5 y $6^{10}$, así que las variaciones en los precios (Var\%) del estrato 6 son idénticas a las del estrato 5. Estas variaciones de precios se hacen con respecto al precio real de diciembre de 2009 y se muestran en las columnas VI, IX y XII. El aumento en precio real del agua para los estratos 5 y 6 es tal que el déficit del sistema permanece constante. Estos aumentos en el precio real generan declinaciones en la demanda que son evaluadas mediante las ecuaciones de demanda estimadas para los mismos estratos. Sin embargo, como las demandas son inelásticas al precio $(-12,9 \%$ y $-11.7 \%$ para los estratos 5 y 6 respectivamente) el recaudo aumenta, y esto permite compensar la caída en los recaudos de los estratos 1 y 2 , generado por la gratuidad del mínimo vital.

Tabla 7. Consumos promedio estratos 1- 4

\begin{tabular}{|c|c|c|c|c|}
\hline \multirow[t]{2}{*}{ Ciudad } & \multicolumn{4}{|c|}{$\begin{array}{l}\text { Consumo promedio por estrato (en m3/hogar } \\
\text { al mes) } \\
\text { Periodo: Enero del año } 2003 \text { a Diciembre } \\
\text { del año } 2008\end{array}$} \\
\hline & 1 & 2 & 3 & 4 \\
\hline Barranquilla & 16,34 & 18,41 & 20,27 & 22,34 \\
\hline Bogotá & 23,90 & 24,75 & 23,58 & 24,20 \\
\hline Bucaramanga & 18,37 & 20,04 & 19,40 & 19,03 \\
\hline Cali & 18,90 & 20,22 & 19,52 & 19,47 \\
\hline Cartagena & 11,53 & 15,83 & 17,44 & 21,75 \\
\hline Cúcuta & 14,10 & 16,24 & 19,72 & 24,68 \\
\hline Ibagué & 14,74 & 16,13 & 16,83 & 16,99 \\
\hline Manizales & 15,16 & 15,79 & 17,24 & 14,93 \\
\hline Medellín & 13,45 & 14,29 & 15,32 & 17,07 \\
\hline Montería & 13,37 & 18,31 & 21,02 & 22,93 \\
\hline Neiva & 16,67 & 19,57 & 20,39 & 22,81 \\
\hline Popayán & 12,79 & 14,96 & 16,85 & 18,68 \\
\hline Santa Marta & 11,75 & 12,26 & 13,90 & 15,98 \\
\hline
\end{tabular}

Fuente: elaboración propia

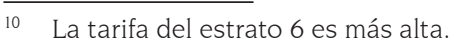


Tabla 8. Cantidad de usuarios por estrato (Enero del año 2010

\begin{tabular}{|l|c|c|c|c|c|c|c|}
\hline \multicolumn{1}{|c|}{ Ciudad } & Estrato 1 & Estrato 2 & Estrato 3 & Estrato 4 & Estrato 5 & Estrato 6 & Total Residencial \\
\hline Barranquilla & 69.261 & 45.049 & 52.640 & 27.273 & 14.010 & 9.322 & 217.555 \\
Bogotá & 46.994 & 229.796 & 277.091 & 140.789 & 50.819 & 41.667 & 787.156 \\
Bucaramanga & 13.474 & 17.706 & 30.064 & 37.457 & 3.769 & 7.737 & 110.207 \\
Cali & 77.065 & 128.162 & 160.515 & 53.053 & 44.740 & 12.350 & 475.885 \\
Cartagena & 63.716 & 49.054 & 31.500 & 11.410 & 6.446 & 7.867 & 169.993 \\
Cúcuta & 30.442 & 51.684 & 29.373 & 13.077 & 2.420 & 409 & 127.405 \\
Ibagué & 12.738 & 42.852 & 27.280 & 11.282 & 1.722 & 539 & 96.413 \\
Manizales & 6.643 & 19.383 & 30.533 & 13.348 & 5.210 & 7.215 & 82.332 \\
Medellín & 45.879 & 186.187 & 179.512 & 72.441 & 55.536 & 28.554 & 568.109 \\
Montería & 33.620 & 13.373 & 6.711 & 2.575 & 1.519 & 964 & 58.762 \\
Neiva & 16.997 & 43.174 & 9.132 & 4.843 & 941 & 190 & 75.277 \\
Popayán & 17.438 & 19.641 & 7.027 & 2.827 & 559 & 56.800 & 104.292 \\
Santa Marta & 9.311 & 17.091 & 25.683 & 7.277 & 3.148 & 7.601 & 70.111 \\
\hline
\end{tabular}

Fuente: elaboración propia

Tabla 9. Porcentaje de beneficiarios, subsidiadores y su relación (enero del año 2010)

\begin{tabular}{|l|c|c|c|}
\hline \multicolumn{1}{|c|}{ Ciudad } & Estrato 1+2 & Estrato 5+6 & Relación \\
\hline Barranquilla & $53 \%$ & $11 \%$ & 4,9 \\
Bogotá & $35 \%$ & $12 \%$ & 3,0 \\
Bucaramanga & $28 \%$ & $10 \%$ & 2,7 \\
Cali & $43 \%$ & $12 \%$ & 3,6 \\
Cartagena & $66 \%$ & $8 \%$ & 7,9 \\
Cúcuta & $64 \%$ & $2 \%$ & 29,0 \\
Ibagué & $58 \%$ & $2 \%$ & 24,6 \\
\hline
\end{tabular}

\begin{tabular}{|l|c|c|c|}
\hline \multicolumn{1}{|c|}{ Ciudad } & Estrato 1+2 & Estrato 5+6 & Relación \\
\hline Manizales & $32 \%$ & $15 \%$ & 2,1 \\
Medellín & $41 \%$ & $15 \%$ & 2,8 \\
Montería & $80 \%$ & $4 \%$ & 18,9 \\
Neiva & $80 \%$ & $2 \%$ & 53,2 \\
Popayán & $36 \%$ & $55 \%$ & 0,6 \\
Santa Marta & $38 \%$ & $15 \%$ & 2,5 \\
\hline
\end{tabular}

Fuente: elaboración propia.

Tabla 10. Resultados de la simulación de la propuesta

\begin{tabular}{|c|c|c|c|c|c|c|c|c|c|c|c|}
\hline & & & \multirow{2}{*}{\multicolumn{9}{|c|}{ Mínimo Vital }} \\
\hline & & & & & & & & & & & \\
\hline & \multicolumn{2}{|c|}{ Precio Dic-2009 } & \multicolumn{3}{|c|}{$10 \mathrm{m3}$} & \multicolumn{3}{|c|}{$8 m 3$} & \multicolumn{3}{|c|}{$6 \mathrm{m3}$} \\
\hline & (III) & (IV) & $(\mathrm{V})$ & (VI) & (VII) & (VIII) & (IX) & $(\mathrm{X})$ & (XI) & (XII) & (XIII) \\
\hline Ciudad & Estrato 5 & $\begin{array}{c}\% \text { del } \\
\text { precio de } \\
\text { Bogotá }\end{array}$ & Precio & Var\% & $\begin{array}{c}\text { \% del } \\
\text { precio de } \\
\text { Bogotá }\end{array}$ & Precio & Var\% & $\begin{array}{c}\text { \% del } \\
\text { precio de } \\
\text { Bogotá }\end{array}$ & Precio & Var\% & $\begin{array}{c}\% \text { del } \\
\text { precio de } \\
\text { Bogotá }\end{array}$ \\
\hline Barranquilla & $1.691,4$ & $70 \%$ & 2.810 & $66,2 \%$ & $84,9 \%$ & 2579 & $52,5 \%$ & $82,5 \%$ & 2350 & $39,0 \%$ & $79,8 \%$ \\
\hline Bogotá & $2.410,2$ & $100 \%$ & 3.311 & $37,4 \%$ & $100,0 \%$ & 3127 & $29,7 \%$ & $100,0 \%$ & 2944 & $22,2 \%$ & $100,0 \%$ \\
\hline Bucaramanga & $1.310,4$ & $54 \%$ & 2.054 & $56,8 \%$ & $62,0 \%$ & 1901 & $45,1 \%$ & $60,8 \%$ & 1749 & $33,5 \%$ & $59,4 \%$ \\
\hline Cali & $1.282,3$ & $53 \%$ & 1.990 & $55,2 \%$ & $60,1 \%$ & 1844 & $43,8 \%$ & $59,0 \%$ & 1700 & $32,6 \%$ & $57,7 \%$ \\
\hline Cartagena & $1.450,9$ & $60 \%$ & 3.598 & $148,0 \%$ & $108,7 \%$ & 3144 & $116,7 \%$ & $100,5 \%$ & 2700 & $86,1 \%$ & $91,7 \%$ \\
\hline Cúcuta & $1.101,9$ & $46 \%$ & 6.067 & $450,7 \%$ & $183,2 \%$ & 4984 & $\mid 352,3 \%$ & $159,4 \%$ & 3935 & $257,1 \%$ & $133,7 \%$ \\
\hline
\end{tabular}


Tabla 10. Resultados de la simulación de la propuesta (continuación)

\begin{tabular}{|c|c|c|c|c|c|c|c|c|c|c|c|}
\hline & & & & & & & Mínimo & ital & & & \\
\hline & Precio D & ic-2009 & & $10 \mathrm{~m} 3$ & & & $8 \mathrm{m3}$ & & & $6 \mathrm{m3}$ & \\
\hline & (III) & (IV) & $(\mathrm{V})$ & (VI) & (VII) & (VIII) & (IX) & (X) & (XI) & (XII) & (XIII) \\
\hline Ciudad & Estrato 5 & $\begin{array}{c}\% \text { del } \\
\text { precio de } \\
\text { Bogotá }\end{array}$ & Precio & Var\% & $\begin{array}{c}\% \text { del } \\
\text { precio de } \\
\text { Bogotá }\end{array}$ & Precio & Var\% & $\begin{array}{c}\text { \% del } \\
\text { precio de } \\
\text { Bogotá }\end{array}$ & Precio & Var\% & $\begin{array}{c}\% \text { del } \\
\text { precio de } \\
\text { Bogotá }\end{array}$ \\
\hline Ibagué & 799,4 & $33 \%$ & 5.730 & $618,8 \%$ & $173,1 \%$ & 4653 & $482,0 \%$ & $148,8 \%$ & 3609 & $351,5 \%$ & $122,6 \%$ \\
\hline Manizales & $1.242,0$ & $52 \%$ & 1.905 & $53,4 \%$ & $57,5 \%$ & 1768 & $42,4 \%$ & $56,5 \%$ & 1633 & $31,5 \%$ & $55,5 \%$ \\
\hline Medellín & $1.061,8$ & $44 \%$ & 1.628 & $53,4 \%$ & $49,2 \%$ & 1511 & $42,4 \%$ & $48,3 \%$ & 1396 & $31,5 \%$ & $47,4 \%$ \\
\hline Montería & $1.177,7$ & $49 \%$ & 4.165 & $253,7 \%$ & $125,8 \%$ & 3523 & $199,2 \%$ & $112,7 \%$ & 2898 & $146,1 \%$ & $98,4 \%$ \\
\hline Neiva & 899,7 & $37 \%$ & 10.770 & $1097,1 \%$ & $325,3 \%$ & 8585 & $854,2 \%$ & $274,5 \%$ & 6474 & $619,6 \%$ & $219,9 \%$ \\
\hline Popayán & 699,2 & $29 \%$ & 2.266 & $224,2 \%$ & $68,4 \%$ & 1930 & $176,1 \%$ & $61,7 \%$ & 1603 & $129,4 \%$ & $54,4 \%$ \\
\hline Santa Marta & $1.348,4$ & $56 \%$ & 2.089 & $55,0 \%$ & $63,1 \%$ & 1936 & $43,6 \%$ & $61,9 \%$ & 1785 & $32,4 \%$ & $60,6 \%$ \\
\hline
\end{tabular}

Fuente: elaboración propia

La tabla 10 revela que el precio real más alto en diciembre de 2009 para el estrato 5 corresponde al de Bogotá. El segundo precio más alto es el de Barranquilla, que es apenas el 70\% del precio de Bogotá. El precio más bajo es el de Popayán, que es menos de una tercera parte del precio de Bogotá. De las columnas VI, IX y XII se concluye que es en Bogotá, en donde se requieren los menores aumentos en las tarifas (37.4\%, 29.7\%, 22\%), para la viabilidad de la propuesta del mínimo vital (de $10 \mathrm{~m}^{3}, 8 \mathrm{~m}^{3}$ y $6 \mathrm{~m}^{3}$ ). Le siguen las ciudades de Manizales, Medellín, Cali, Bucaramanga y Santa Marta.

Neiva es una ciudad donde resulta inviable la propuesta, ya que requeriría aumentos en las tarifas de más de seis veces el precio real inicial para $6 \mathrm{~m}^{3}$, hasta más de diez veces para $10 \mathrm{~m}^{3}$. Otras ciudades con muy poca viabilidad de aplicación del mínimo vital son: Ibagué, que requeriría aumentos en las tarifas de tres y medio veces el precio real inicial para $6 \mathrm{~m}^{3}$ hasta más de seis veces para 10 $\mathrm{m}^{3}$ y Cúcuta, con aumentos en las tarifas de más de dos y medio veces el precio real inicial para 6 $\mathrm{m}^{3}$ hasta más de cuatro y medio veces para $10 \mathrm{~m}^{3}$. En las ciudades de Popayán, Cartagena y Montería la propuesta tal vez solo sería factible para un mínimo vital de $6 \mathrm{~m}^{3}$, que aunque requeriría de aumentos aproximados al 100\% y hasta un poco más, la tarifa no sería tan alta, porque seguiría por debajo del precio de Bogotá, especialmente Popayán, cuya tarifa sería solo ligeramente superior a la mitad de la de Bogotá.

Como es lógico, el ranking de viabilidad depende de la relación entre beneficiarios y subsidiadores que aparece en la tabla 9. Las ciudades que presentan la relación más alta de beneficiadores sobre subsidiadores son las mismas en las que resulta inviable la propuesta: Neiva, Ibagué y Cúcuta.

\section{CONCLUSIONES}

El mínimo vital de agua potable es la cantidad mínima de agua que debe utilizar cada persona para tener unas condiciones de vida digna, para cubrir los requerimientos básicos de higiene y consumo, las necesidades vitales como bañarse y cocinar. No hay una medida universal para el mínimo vital; esta varía de acuerdo con las diferencias culturales, climáticas, sociales y económicas entre las regiones del mundo. Por tanto, se debe estudiar cada región antes de tratar de establecerlo. 
En ONU (2010) la Asamblea General de las Naciones Unidas declaró el derecho al agua potable y el saneamiento, como derecho humano. Sin embargo, esto no convirtió al agua en un derecho fundamental de los colombianos, tutelable y exigible, porque no es vinculante.

El referendo del agua propuesto en Colombia tenía como propósito lograr que en la Constitución, quedara consagrado el acceso al agua como un derecho fundamental, así todas las personas tendrían la posibilidad de un suministro mínimo en condiciones gratuitas. Sin embargo, desde la perspectiva económica la gratuidad universal no es necesaria ni conveniente. La gratuidad puede ser necesaria para los abonados con mayores dificultades de pago por su escaso ingreso, pero innecesaria para abonados de estratos altos que por su capacidad de pago tienen garantizado el acceso al mínimo vital. Resultaría, además, contraproducente en Colombia, porque erosionaría el recaudo con el que se financian los subsidios de las tarifas de los usuarios con mayores carencias.

La revisión bibliográfica y los resultados de las estimaciones de las funciones de demanda de agua coinciden, en que el valor absoluto de la elasticidad precio de la demanda aumenta a medida que aumenta el estrato. Esto se debe a que los usuarios de los estratos más bajos están sobre su consumo básico y no tienen margen de reacción ante el incremento de los precios. Sin embargo, a diferencia de las otras investigaciones, esta encontró que las elasticidades precio de la demanda para los estratos uno hasta cuatro es cero, así que el margen de reacción estimado es nulo.

La simulación de la propuesta del mínimo vital de agua potable en Colombia, permitió evaluar la viabilidad de su implementación, y se encontró que existen ciudades como Neiva, Ibagué y Cúcuta en donde el otorgamiento del mínimo vital a los estratos uno y dos no podría ser subsidiado por los estratos 5 y 6 , y se necesitaría el financiamiento externo al sistema. En contraste, ciudades como
Bogotá, Manizales, Medellín, Cali, Bucaramanga y Santa Marta, son en su orden, las que presentan mayor viabilidad. En las ciudades de Popayán, Cartagena y Montería la propuesta tal vez solo sería factible para un mínimo vital de seis metros cúbicos a lo sumo.

Se observó que el ranking de viabilidad depende de la relación entre número de beneficiarios y subsidiadores, porque las ciudades que presentan la relación más alta de beneficiadores sobre subsidiadores son las mismas en las que resulta inviable la propuesta.

\section{BIBLIOGRAFÍA}

Alfaro, Y.; Espeleta, J.; Fontalvo, L. y García, B. (2009). Consecuencias jurídicas y sociales que traería para Colombia la aprobación del referendo del agua. Monografía presentada como requisito para obtener el título de Abogado. Universidad Simón Bolívar, Barranquilla.

Araujo, A. (2009). Agua: derecho humano fundamental. En: Revista Jurídica UNIGRAN, Vol. 11, N²2, p. 11-21.

CRC - Congreso de la República de Colombia- (2008). Proyecto de ley número 171. Por medio de la cual se convoca a un Referendo Constitucional para consagrar el derecho al agua potable como fundamental y otras normas concordantes. Bogotá, Colombia.

CRC (1994). Ley Número 142. Por la cual se establece el régimen de los servicios públicos domiciliarios y se dictan otras disposiciones. Diario Oficial N $N^{\circ} 41.433$ de 11 de julio.

Convocatoria Referendo por el Agua (2007). Pronunciamientos de Organizaciones Gremiales. Unidad nacional por el agua como bien común y derecho fundamental. Documento de Trabajo.

CRA - Comisión de Regulación de Agua Potable y Saneamiento Básico- (2008). Consumo Básico o de Subsistencia en el Servicio de Acueducto y Alcantarillado. Colombia

Davidson A. (2009). Control y manejo local de nuestras aguas como bien común Luchas ejemplares y desafíos. Fundación Heinrich Böll Stiftung, The Council of Canadians $y$ Our Water Commons, 68p. 
Díaz, A.; Chingate, N.; Muñoz, D.; Olaya, W.; Perilla, C.; Sánchez, F. y Sánchez, K. (2009). Desarrollo sostenible y el agua como derecho en Colombia. En: Estudios socio-jurídicos, Vol. 11, $\mathrm{N}^{\circ} 1$, p. 84-116.

Escobar, A. (2009). Implicaciones del referendo por el agua. [En líneal Portafolio.com, 31 de marzo. Disponible en: http://www.portafolio.co/archivo/documento/CMS-4929752. [Consultado 15 de junio de 2010].

Hewitt, J. y Hanemann, M. (1995). A Discrete Continuous Approach to Residential Water Demand Under Block Rate Pricing. En: Land Economics, Col. 71, N 2, p. 173-192.

Horward, G. y Bartram, J. (2003). Domestic Water Quantity, Service Level and Health. World Health Organization, 39p.

Junca, J. (2000). Determinación del consumo básico de agua potable subsidiable en Colombia. Archivos de Macroeconomía, Departamento Nacional de Planeación, Documento 139, 61p.

Martínez A. (2004). Sin empresas ni gobiernos. El Fórum Social Mundial de 2004 (Crónica de un viaje a la India). En: Ecología Política, $N^{\circ} 27$, p. 24-25.

Medina, C. y Morales, L. (2007). Demanda por servicios públicos domiciliarios en Colombia y subsidios: implicaciones sobre el bienestar. Borradores de Economía, 004293, Banco de la República, No 467, noviembre, 38p.

Marín, L. (2009). Mínimo vital de agua en Medellín: un impulso al Referendo del Agua. [En línea] La Silla vacía. Disponible en: http://www.lasillavacia.com/ historia/2329. [consultado 10 de junio de 2010].
Muñoz, O. (2009). El derecho al agua potable como derecho fundamental no enumerado. Derechos constitucionales no escritos reconocidos por el Tribunal Constitucional, Lima, Gaceta Jurídica, p. 169-180.

ONU -Organización de las Naciones Unidas- (2010). Resolución sobre derecho humano al agua y el saneamiento. A/64/L.63/Rev.1

PNUD -Programa de las Naciones Unidas para el Desarrollo- (2006). Informe sobre Desarrollo Humano 2006.

Smets, H. (2006a). Por un derecho efectivo al agua. Universidad Colegio Mayor de Nuestra Señora del Rosario, Facultad de Jurisprudencia, Bogotá, septiembre, 180p.

Smets, H. (2006b). El derecho al agua en las legislaciones nacionales. Universidad Colegio Mayor de Nuestra Señora del Rosario, Facultad de Jurisprudencia, Bogotá, septiembre, 112p.

Suarez, A. y Cardona, I. (2008). Referendo constitucional del Agua: Sustentación Económica del Mínimo Vital gratuito. [En línea] Disponible en: http://www. ecofondo.org.co/ecofondo/dmdocuments/MINIMO\%20VITAL\%20GRATUITO.pdf. [Consultado 18 de Mayo de 2010].

Valencia, G. (2008). La propuesta de un mínimo vital de agua en Colombia. En: Debates (Medellín), Universidad de Antioquia, $N^{\circ} 48$, p. 50-55. 
ANEXO A. Evolución del consumo promedio de agua para cada ciudad y por estrato.
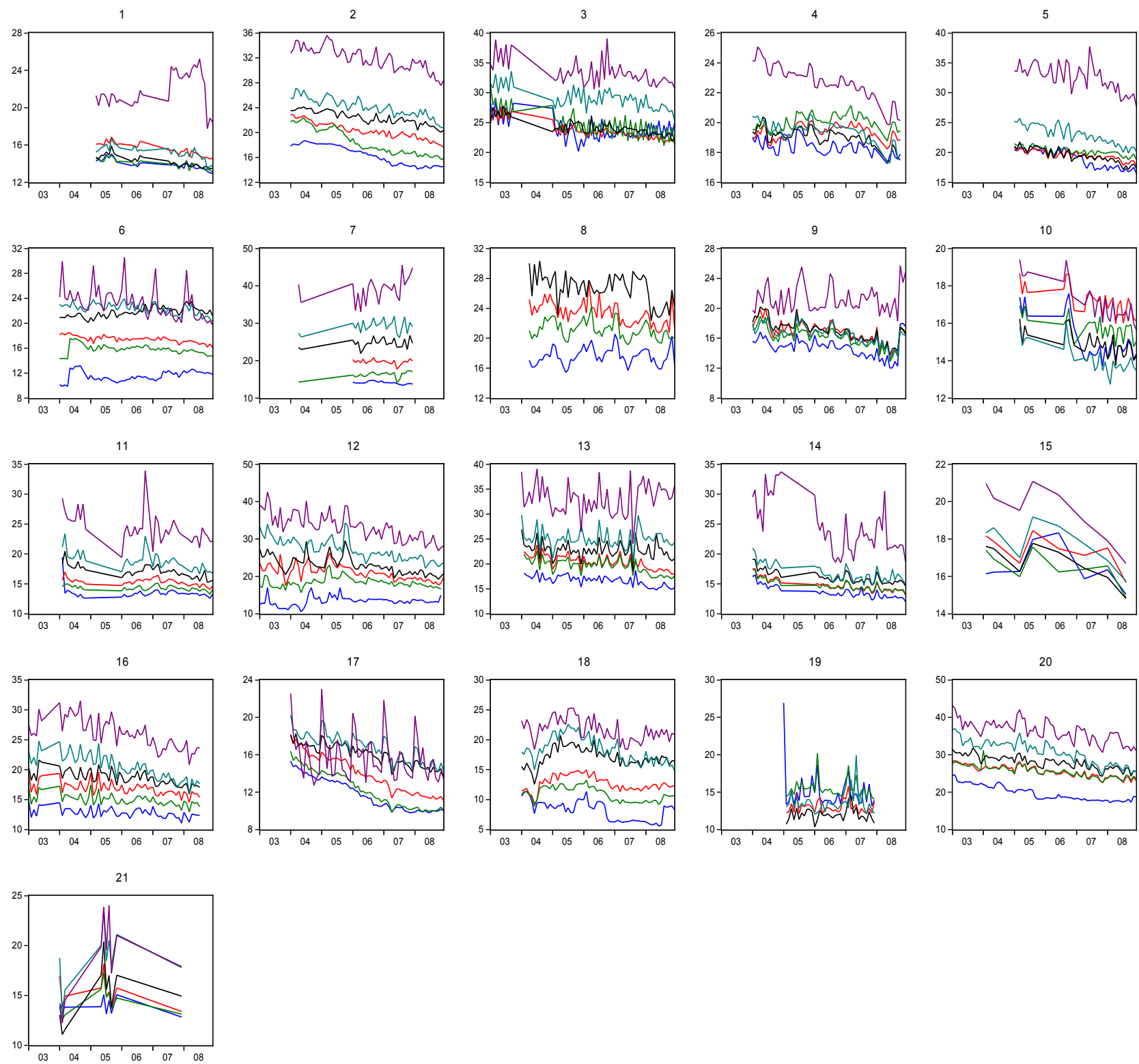

$\overline{ }_{\mathrm{C} 4}^{\mathrm{C} 1} \overline{\mathrm{C}}_{\mathrm{C5}}^{\mathrm{C}} \overline{\mathrm{C}}_{\mathrm{C}}^{\mathrm{C}}$ 
ANEXO B. Componente de heterogeneidad individual para cada uno de los seis estratos

\begin{tabular}{|c|c|c|c|c|c|c|}
\hline & \multicolumn{6}{|c|}{ Estrato } \\
\hline Ciudad & 1 & 2 & 3 & 4 & 5 & 6 \\
\hline Armenia & $-1,354$ & $-3,572$ & $-2,925$ & $-5,866$ & $-0,330$ & $-0,182$ \\
\hline Barranquilla & 1,067 & 0,841 & 1,762 & 2,206 & 0,203 & 0,250 \\
\hline Bogotá & 8,621 & 7,177 & 5,079 & 4,072 & 0,443 & 0,349 \\
\hline Bucaramanga & 3,093 & 2,475 & 0,899 & $-1,098$ & $-0,066$ & $-0,130$ \\
\hline Cali & 3,627 & 2,655 & 1,018 & $-0,666$ & 0,142 & 0,270 \\
\hline Cartagena & $-3,747$ & $-1,736$ & $-1,068$ & 1,618 & 0,140 & $-0,050$ \\
\hline Cúcuta & $-1,178$ & $-1,329$ & 1,218 & 4,545 & 0,383 & 0,438 \\
\hline Florencia & 2,321 & 3,568 & 4,839 & 6,582 & sin infor. & sin infor. \\
\hline Ibagué & $-0,540$ & $-1,435$ & $-1,671$ & $-3,140$ & $-0,295$ & $-0,256$ \\
\hline Manizales & $-0,112$ & $-1,783$ & $-1,261$ & $-5,198$ & $-0,351$ & $-0,379$ \\
\hline Medellín & $-1,828$ & $-3,281$ & $-3,181$ & $-3,063$ & $-0,067$ & $-0,035$ \\
\hline Montería & $-1,909$ & 0,742 & 2,520 & 2,798 & 0,296 & 0,271 \\
\hline Neiva & 1,390 & 1,998 & 1,890 & 2,683 & 0,139 & 0,193 \\
\hline Pasto & $-1,666$ & $-2,869$ & $-3,726$ & $-4,023$ & sin infor. & $-0,029$ \\
\hline Pereira & 1,250 & $-1,077$ & $-1,127$ & $-3,432$ & $-0,160$ & sin infor. \\
\hline Popayán & $-2,491$ & $-2,606$ & $-1,657$ & $-1,450$ & $-0,083$ & $-0,059$ \\
\hline Santa Marta & $-3,524$ & $-5,306$ & $-4,601$ & $-4,147$ & $-0,177$ & $-0,483$ \\
\hline Sincelejo & $-7,109$ & $-6,655$ & $-5,767$ & $-3,044$ & $-0,065$ & $-0,125$ \\
\hline Tunja & $-0,313$ & $-2,133$ & $-5,493$ & $-8,152$ & $-0,375$ & sin infor. \\
\hline Valledupar & 4,385 & 7,994 & 7,053 & 7,881 & 0,340 & 0,289 \\
\hline Villavicencio & $-1,359$ & $-3,172$ & $-3,245$ & $-4,982$ & $-0,117$ & $-0,331$ \\
\hline
\end{tabular}

Fuente: elaboración propia 